\title{
ON A COMPLEMENT TO VALIRON'S TAUBERIAN THEOREM FOR THE STIELTJES TRANSFORM
}

\author{
DANIEL F. SHEA ${ }^{1}$
}

One of the classical abelian-tauberian theorems, due to Valiron [14], is

THEOREM A. Let $\psi$ be an increasing function such that $\psi(0)=0$ and

$$
F(x)=\int_{0}^{\infty} \frac{d \psi(t)}{x+t}
$$

converges for $x>0$. Then

$$
\psi(t) \sim t^{\lambda} L(t) \quad(0 \leqq \lambda<1, t \rightarrow \infty),
$$

where $L$ is a slowly-varying function, if and only if ${ }^{2}$

$$
F(x) \sim(\pi \lambda / \sin \pi \lambda) x^{\lambda-1} L(x) \quad(x \rightarrow \infty) .
$$

The term "slowly-varying" here is used in the sense of Karamata [8], and means that $L$ is positive and satisfies

$$
L(\sigma t) / L(t) \rightarrow 1 \quad(t \rightarrow \infty)
$$

for every $\sigma>0$.

The relation between Theorem A, stated in terms of slowly-varying functions $L$, and Valiron's original form of the theorem which uses the notion of "proximate orders," is explained in [7].

Feller [5, p. 419] has pointed out the importance of observing that in such an abelian-tauberian statement, the relations (2) and (3) are equivalent to

$$
\lim _{t \rightarrow \infty} \frac{\psi(\sigma t)}{t F(t)}=\frac{\sin \pi \lambda}{\pi \lambda} \sigma^{\lambda} \quad(0<\sigma<\infty) .
$$

That each of (2) and (3) implies (4) is the content of Theorem A, a standard tauberian theorem of some depth (cf. [12], [6]). The converse assertion, that (4) implies (2) and (3), is on the other hand quite obvious: for example, (4) implies that $\psi(\sigma t) / \psi(t) \rightarrow \sigma^{\lambda}(t \rightarrow \infty)$ for every $\sigma>0$, and this is clearly equivalent to (2). However, the following theorem shows that the truth of (4) for a single value of $\sigma(\sigma=1$, say) is already sufficient to imply (2) and (3).

Received by the editors August 22, 1967 and, in revised form, November 20, 1967.

1 Research supported in part by NSF grant GP-5728.

2 When $\lambda=0, \pi \lambda / \sin \pi \lambda$ is to be replaced by its limit, $=1$. 
TheOREM B. Let $\psi$ and $F$ be as defined in Theorem A. Then the condition

$$
\alpha=\lim _{t \rightarrow \infty} \frac{\psi(t)}{t F(t)} \quad \text { exists } \quad(\alpha>0)
$$

implies $\alpha \leqq 1$, and (2) and (3) are true with $\lambda$ determined by

$$
\alpha=\sin \pi \lambda / \pi \lambda \quad(0 \leqq \lambda<1) .
$$

Although Theorem A (as well as many similar tauberian theorems) is often presented with conditions on $L$ other than (K), part of the significance of Theorem B is that it justifies Karamata's condition as the most natural one. (In this connection, cf. also Feller's discussion of slow variation in $[4$, p. 317].)

An early form of Theorem B appeared in my thesis (cf. [11, Corollary 1.1] for the statement and proof of an analogous result); however, that result was not sharp since I proved there only that (5) implies (2) and (3) with

$$
L(t)=t^{\epsilon(t)} \quad(\epsilon(t) \rightarrow 0 \text { as } t \rightarrow \infty)
$$

in place of $(\mathrm{K})$.

More recently, Edrei and Fuchs [3] established the sharp form of Theorem $\mathrm{B}$ given above, together with a similar result involving a kernel different from the one in (1). Their proofs use an impressive array of ingenious and powerful techniques developed in [3] as well as in some of their earlier joint work.

Still more recently, Drasin [1] has succeeded in extending the method of Edrei and Fuchs to cover a wide class of convolution transforms

$$
F(x)=\int_{-\infty}^{\infty} k(x-t) f(t) d t .
$$

Thus if $k$ is a strictly positive $L^{1}(-\infty, \infty)$ kernel behaving suitably at $\pm \infty, f$ increases (or does not decrease too rapidly), and $F$ is defined by (7), then Drasin's theorem asserts that the existence and positivity of $\lim _{x \rightarrow \infty} f(x) / F(x)$ implies $f(x)=e^{\lambda x} \phi(x)$, where $L(t)$ $=\phi(\log t)$ satisfies $(\mathrm{K})$. In particular, this general result contains Theorem B.

It is the purpose of this note to develop a new method of proving Theorem $B$ which is short and quite transparent, and leads immediately to several refinements (Theorems $B^{\prime}$ and $C$, discussed in $\$ 2$ ). The present method is also capable of considerable generalization, 
but I confine myself here to the case of the Stieltjes transform (1) in order to present the main ideas as clearly as possible.

The proof of Theorem B to be given here also throws some light on the essential features of the "tauberian" (for lack of a better term) hypothesis (5). In particular, the first part of the proof involves reducing the problem to that of solving a certain integral equation; this in itself is typical of tauberian theorems (compare Wiener's remarks in $[16$, p. 50]). But a comparison of the difficulties encountered in dealing with the trivial integral equation corresponding to the classical theorem $(3) \Rightarrow(2)$, and those involved here in proving (5) $\Rightarrow(2)$, suggests that in fact Theorem B lies deeper than Theorem A.

One further remark: the statement, as well as the present proof, of Theorem B contrast interestingly with the Paley-Wiener generalization of Mercer's theorem [10], especially in the dependence of this proof on the location of the "ones" of the Fourier transform of the kernel in (1).

1. Proof of Theorem B. An integration by parts transforms (1) into

$$
F(x)=\int_{0}^{\infty} \frac{\psi(t)}{(t+x)^{2}} d t
$$

in view of the following simple remark (which we shall have occasion to use again): If $\phi$ is a positive, increasing function such that

$$
\int_{1}^{\infty} \frac{d \phi(t)}{t}<\infty \text { or } \int_{1}^{\infty} \frac{\phi(t)}{t^{2}} d t<\infty
$$

then

$$
\phi(t)=o(t) \quad(t \rightarrow \infty) .
$$

It is clear from (1.1) that $F(u x) \leqq F(x)$ for $u \geqq 1$. This observation together with (5) shows that

$$
1 \leqq \liminf _{t \rightarrow \infty} \frac{\psi(u t)}{\psi(t)} \leqq \limsup _{t \rightarrow \infty} \frac{\psi(u t)}{\psi(t)} \leqq u
$$

for all $u \geqq 1$. Fix $\sigma>1$, and choose any sequence $t_{n} \rightarrow \infty$ such that

$$
C=\lim _{n \rightarrow \infty} \frac{\psi\left(\sigma t_{n}\right)}{\psi\left(t_{n}\right)}
$$

exists. Then the functions $g_{n}(u)=\psi\left(u t_{n}\right) / \psi\left(t_{n}\right)$ clearly increase and are uniformly bounded on any finite interval. 
Applying the "selection principle" [15] successively on the intervals $0 \leqq u \leqq u_{0}\left(u_{0}=1,2, \cdots\right)$ in a standard way, we find a subsequence $n_{k}$ such that $g_{n_{k}}$ converges for all $u \geqq 0$ to an increasing function $g$, with

$$
g(1)=1, \quad g(\sigma)=C .
$$

Making a change of variables in (1.1) leads to

$$
\frac{x \tau_{k} F\left(x \tau_{k}\right)}{\psi\left(x \tau_{k}\right)} \frac{\psi\left(x \tau_{k}\right)}{\psi\left(\tau_{k}\right)}=x \int_{0}^{\infty} \frac{\psi\left(u \tau_{k}\right)}{\psi\left(\tau_{k}\right)} \frac{d u}{(u+x)^{2}} \quad\left(\tau_{k}=t_{n_{k}}\right)
$$

for any $x>0$ and all sufficiently large $k$. By Fatou's lemma, and (5), we deduce

$$
g(x) \geqq \alpha x \int_{0}^{\infty} \frac{g(u)}{(u+x)^{2}} d u .
$$

In fact, equality holds in (1.6). To see this, we use the estimate

$$
F(r)<\int_{0}^{R} \frac{\psi(t)}{(t+r)^{2}} d t+4 F(R) \quad(r>0, R>0),
$$

an immediate consequence of the simple relations

$$
\int_{R}^{\infty} \frac{\psi(t)}{(t+r)^{2}} d t<\int_{R}^{\infty} \frac{\psi(t)}{t^{2}} d t<4 \int_{R}^{\infty} \frac{\psi(t)}{(t+R)^{2}} d t \leqq 4 F(R) .
$$

In (1.7) set $r=x \tau_{k}$ and $R=s x \tau_{k}$, where $x>0, s>0$; then the left side of (1.5) is dominated by

$$
x \int_{0}^{s x} \frac{\psi\left(u \tau_{k}\right)}{\psi\left(\tau_{k}\right)} \frac{d u}{(u+x)^{2}}+\left(\frac{4}{s}\right) \frac{s x \tau_{k} F\left(s x \tau_{k}\right)}{\psi\left(s x \tau_{k}\right)} \frac{\psi\left(s x \tau_{k}\right)}{\psi\left(\tau_{k}\right)}
$$

for all sufficiently large $k$. Letting $k \rightarrow \infty$, we use (5) again, and bounded convergence, to deduce

$$
g(x) \leqq \alpha x \int_{0}^{s x} \frac{g(u)}{(u+x)^{2}} d u+\frac{4}{s} g(s x) .
$$

Now let $s \rightarrow \infty$, and use (A) and (1.6) to obtain finally

$$
g(x)=\alpha x \int_{0}^{\infty} \frac{g(u)}{(u+x)^{2}} d u \quad(x>0) .
$$

A solution of this equation which increases and satisfies $g(1)=1$ is given by $g(x)=x^{\lambda}$, with $\lambda$ determined by (6). If this were the only 
such solution, then we would have necessarily $C=\sigma^{\lambda}$, by (1.4), and thus a comparison with (1.3) yields

$$
\lim _{t \rightarrow \infty} \frac{\psi(\sigma t)}{\psi(t)}=\sigma^{\lambda} \quad(0<\sigma<\infty),
$$

since in the above argument $\sigma(>1)$ was arbitrary. But (1.9) is obviously equivalent to (2).

To complete the proof of Theorem B, then, it is enough to show that the only admissible (i.e. increasing, positive) solutions of (1.8) are given by

$$
g(x)=A x^{\lambda} \quad(A>0),
$$

where $\lambda$ satisfies (6).

We first show that

$$
\beta=\limsup _{x \rightarrow \infty} \frac{\log g(x)}{\log x}<1
$$

holds for any admissible solution $g$ of (1.8). Indeed, it is obvious from (1.8) and (A) that $\beta \leqq 1$, and if $\beta=1$, then an elementary argument $\left[11\right.$, p. 208] shows that there exist sequences $x_{n}, S_{n}$ such that

$$
x_{n} \rightarrow \infty, S_{n} / x_{n} \rightarrow \infty \quad(n \rightarrow \infty)
$$

and

$$
g(u) \geqq(1+o(1))\left(g\left(x_{n}\right) / x_{n}\right) u \quad\left(x_{n} \leqq u \leqq S_{n}, \text { to } n \rightarrow \infty\right) .
$$

Using this inequality in the estimand (1.6) yields

$$
g\left(x_{n}\right) \geqq \alpha(1+o(1)) g\left(x_{n}\right) \int_{x_{n}}^{S_{n}} \frac{u d u}{\left(u+x_{n}\right)^{2}} \quad(n \rightarrow \infty)
$$

and hence

$$
1 \geqq \frac{\alpha}{2} \int_{1}^{s_{n} / z_{n}} \frac{t d t}{(t+1)^{2}} \quad\left(n \geqq n_{0}\right) .
$$

This inequality is impossible in view of (1.12) and the fact that $\alpha>0$, and thus (1.11) is true.

Making an exponential change of variable, equation (1.8) becomes

$$
G(y)=\int_{-\infty}^{\infty} G(t) k(y-t) d t \quad\left(G(y)=g\left(e^{y}\right)\right),
$$

with 


$$
k(x)=\alpha\left(e^{x / 2}+e^{-x / 2}\right)^{-2} .
$$

A standard result in Fourier analysis [13, p. 305] asserts that any solution of (1.13) such that

$$
G(y)=O\left(e^{a|y|}\right) \quad(y \rightarrow \pm \infty)
$$

for some $a<1$ must have the form

$$
G(y)=\sum_{\nu} \sum_{p=1}^{q_{\nu}} A_{\nu, p} y^{p-1} \exp \left[-i \omega_{\nu} y\right]
$$

where $\omega_{\nu}$ runs through the sequence of zeros of $\hat{k}(\omega)-1$ such that $\left|\operatorname{Im} \omega_{\nu}\right| \leqq a$, and where $q_{\nu}$ is the multiplicity of the zero $\omega_{\nu}$, the $A_{\nu, p}$ are constants and

$$
\hat{k}(\omega)=\int_{-\infty}^{\infty} k(x) e^{i x \omega} d x=\alpha \int_{0}^{\infty} \frac{t^{i \omega}}{(t+1)^{2}} d t=\alpha \frac{i \pi \omega}{\sin (i \pi \omega)} .
$$

Clearly, there can only be a finite number of solutions $\omega_{\nu}=\xi_{\nu}+i \eta_{\nu}$ of $\hat{k}(\xi+i \eta)=1$ in $|\eta| \leqq a$, since $\hat{k}(\xi+i \eta) \rightarrow 0$ uniformly in this strip when $\xi \rightarrow \pm \infty$.

Note that by (1.11) any admissible solution $G$ of (1.13) must satisfy (1.14). Further, since $G$ must be real and positive we need consider only the zeros $\omega_{\nu}$ which are purely imaginary, that is the $\omega_{\nu}=i \eta_{\nu}$ such that

$$
\sin \pi \eta_{\nu} / \pi \eta_{\nu}=\alpha \quad\left(-1<\eta_{\nu}<1\right) .
$$

It follows from (1.16) that $\alpha \leqq 1$. If $\alpha<1$, there are precisely two values $\eta_{\nu}$ for which (1.16) is true, call them $\lambda$ and $-\lambda(0<\lambda<1)$. Then (1.15) reduces to

$$
G(y)=A e^{\lambda y}+B e^{-\lambda y}, \quad g(x)=A x^{\lambda}+B x^{-\lambda} .
$$

Since $g$ is increasing and positive for $x>0$, necessarily $A>0$ and $B=0$, which proves (1.10) when $\alpha<1$.

If $\alpha=1$, then a glance at (1.16) and (1.15) shows that in this case

$$
g(x)=A+B \log x,
$$

with $A>0$ and $B=0$. This completes the proof of (1.10), and hence also of Theorem B.

2. Further remarks on Theorem B. One additional feature of the above proof should be explicitly pointed out: with only a single notational change (replace " $t_{n} \rightarrow \infty$ " in the second paragraph of $\$ 1$ by " $t_{n} \rightarrow 0$ "), it also yields the following complement which describes the asymptotic behavior of the Stieltjes transform at the origin. 
THEOREM C. The statement of Theorem $\mathrm{B}$ remains true if $\infty$ is replaced by 0 in (5), (2), (3) and (K).

Thus the existence of

$$
\alpha=\lim _{t \rightarrow 0} \frac{\psi(t)}{t F(t)} \quad(\alpha>0)
$$

implies that both $\psi$ and $F$ vary regularly at the origin. The converse statement, that if either $\psi$ or $F$ varies regularly at the origin then so does the other (and hence the limit in (2.1) exists), is due to Hardy and Littlewood [6]. ${ }^{3}$

The motivation for a "ratio-tauberian" theorem for the Stieltjes transform arose from a problem in the value-distribution theory of entire functions, where the asymptotic behavior of ratios such as $N(r, 0) / \log M(r, f)$, as $r \rightarrow \infty$, is of key interest. Here, as in the usual notation of the theory,

$$
M(r, f)=\max _{\theta}\left|f\left(r e^{i \theta}\right)\right|, \quad N(r, 0)=\int_{0}^{r} \frac{n(t, 0)}{t} d t \quad(\text { if } f(0) \neq 0),
$$

and $n(t, 0)$ denotes the number of zeros of the entire function $f$ in the $\operatorname{disk}|z| \leqq t$.

An important role is played in this theory by functions $f$ of the form

$$
f(z)=\prod_{\nu=1}^{\infty}\left(1+\frac{z}{a_{\nu}}\right) \quad\left(0<a_{\nu} \leqq a_{\nu+1}\right),
$$

since functions with negative zeros are "extremal" for many problems. Of particular interest are the "Lindelöf functions," i.e. functions of the form (2.2) with zeros $-a_{\nu}$ distributed so regularly that

$$
N(r, 0) \sim r^{\lambda} L(r) \quad(0 \leqq \lambda<1, r \rightarrow \infty),
$$

and Valiron's Theorem A asserts that (2.3) is equivalent to

$$
\log M(r, f) \sim(\pi \lambda / \sin \pi \lambda) r^{\lambda} L(r) \quad(r \rightarrow \infty) .
$$

(To see this, just notice that (2.2) implies

$$
\left.\log M(r, f)=\int_{0}^{\infty} \log \left(1+\frac{r}{t}\right) d n(t, 0)=r \int_{0}^{\infty} \frac{d N(t, 0)}{r+t} \cdot\right)
$$

\footnotetext{
${ }^{3}$ Actually, the relevant result in [6, Theorem 4] is stated only for the case $L(t)$ $\equiv$ constant, but the extension to general functions $L$ varying slowly at the origin is an easy exercise.
} 
Thus the Lindelöf functions satisfy

$$
\lim _{r \rightarrow \infty} \frac{N(r, 0)}{\log M(r, f)}=\frac{\sin \pi \lambda}{\pi \lambda}
$$

and the content of Theorem B is that the only functions of the form (2.2) which satisfy (2.5) are the Lindelöf functions.

Finally, we mention a generalization of Theorem B which-as D. Drasin has remarked-follows immediately from the above proof, and which will be convenient to have available for use in a subsequent paper.

Theorem $\mathrm{B}^{\prime}$. Let $\psi$ and $F$ be defined as in Theorem A, and let $G \subset(0, \infty)$ be any set of the form

$$
G=\bigcup_{n=1}^{\infty}\left(a_{n}, b_{n}\right) \quad\left(a_{n} \rightarrow \infty, b_{n} / a_{n} \rightarrow \infty\right) .
$$

\section{If the limit}

$$
\alpha=\lim _{t \rightarrow \infty ; t \in G} \frac{\psi(t)}{t F(t)}
$$

exists and is positive, then $\alpha \leqq 1$ and, if $\lambda$ is determined by (6),

$$
\lim _{n \rightarrow \infty} \frac{\psi\left(\sigma t_{n}\right)}{\psi\left(t_{n}\right)}=\sigma^{\lambda} \quad(0<\sigma<\infty)
$$

holds for every sequence $\left\{t_{n}\right\}$ satisfying the condition

$$
\sigma t_{n} \in G \quad\left(0<\sigma<\infty, n>n_{0}(\sigma)\right) .
$$

"Locally tauberian" theorems, in which conclusions like (2.7) are deduced from hypotheses somewhat different from those in the above statement, have recently been established by Edrei in his interesting paper [2].

\section{REFERENCES}

1. D. Drasin, Tauberian theorems and slowly varying functions, Trans. Amer. Math. Soc. 138 (1968), 333-356.

2. A. Edrei, Locally tauberian theorems for meromorphic functions of lower order less than one, Trans. Amer. Math. Soc. (to appear).

3. A. Edrei and W. H. J. Fuchs, Tauberian theorems for a class of meromorphic functions with negative zeros and positive poles, Proc. Internat. Conf. in Function Theory, Erevan, Armenian S.S.R., 1966.

4. W. Feller, On the classical Tauberian theorems, Arch. Math. 14 (1963), 317-322.

5. - Introduction to probability theory and its applications, Vol. II, Wiley, New York, 1966. 
6. G. H. Hardy and J. E. Littlewood, Notes on the theory of series (XI): On Tauberian theorems, Proc. London Math. Soc. 30 (1930), 23-37.

7. S. Hellerstein and D. F. Shea, Bounds for the deficiencies of mermorphic functions of finite order, Proc. Sympos. Pure Math., Vol. 11, Amer. Math. Soc., Providence, R. I., 1968.

8. J. Karamata, Sur un mode de croissance régulière, Mathematica (Cluj) 4 (1930), 38-53.

9. B. Kjellberg, On the minimum modulus of entire functions of lower order less than one, Math. Scand. 8 (1960), 189-197.

10. R. E. A. C. Paley and N. Wiener, Notes on the theory and application of Fourier transforms VII: On the Volterra equation, Trans. Amer. Math. Soc. 35 (1933), 785791.

11. D. F. Shea, On the Valiron deficiencies of meromorphic functions of finite order, Trans. Amer. Math. Soc. 124 (1966), 201-222.

12. E. C. Titchmarsh, On integral functions with real negative zeros, Proc. London Math. Soc. (2) 26 (1927), 185-200.

13. - Theory of Fourier integrals, Oxford Univ. Press, London, 1937.

14. G. Valiron, Sur les fonctions entières d'ordre fini et d'ordre nul, et en particulier les fonctions a correspondance régulière, Ann. Fac. Sci. Univ. Toulouse (3) 5 (1913), 117-257.

15. D. V. Widder, The Laplace transform, Princeton Univ. Press, Princeton, N. J., 1946.

16. N. Wiener, Tauberian theorems, Ann. of Math. 33 (1932), 1-100.

UNIVERSITY OF WISCONSIN 